\title{
EFFECT OF STOICHIOMETRY ON DEFECT DISTRIBUTION IN CUBIC GaN GROWN ON GaAs BY PLASMA-ASSISTED MBE:-
}

S. RUVIMOV, Z. LILIENTAL-WEBER, and J. WASHBURN, Lawrence Berkeley National Laboratory, Berkeley $94720, \mathrm{CA}$

T. J. DRUMMOND, M. HAFISH, and S. R. LEE,

Sandia National Laboratories, Albuquerque, NM 87185

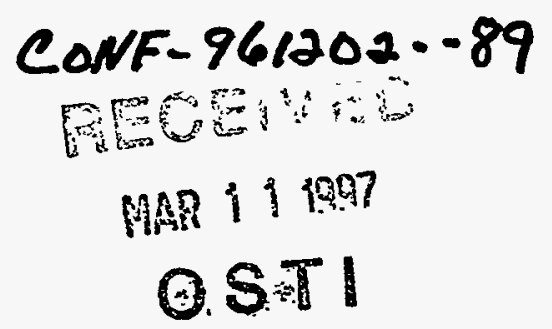

\section{ABSTRACT}

High resolution electron microscopy has been applied to characterize the structure of $\beta$ $\mathrm{GaN}$ epilayers grown on (001) GaAs substrates by plasma-assisted molecular-beam-epitaxy. The rf plasma source was used to promote chemically active nitrogen. The layer quality was shown to depend on growth conditions ( $\mathrm{Ga}$ flux and $\mathrm{N}_{2}$ flow for fixed if power). The best quality of $\mathrm{GaN}$ layers was achieved by "stoichiometric" growth; Ga-rich layers contain a certain amount of the wurtzite phase. GaN layers contain a high density of stacking faults which drastically decreases toward the GaN surface. Stacking faults are anisotropically distributed in the GaN layer, the majority intersect the interface along lines parallel to the "major flat" of the GaAs substrate. This correlates well with the observed anisotropy in the intensity distribution of x-ray reflexions. Formation of stacking faults are often associated with atomic steps at the GaN-GaAs interfaces.

\section{INTRODUCTION}

GaN currently attracts the interest of many researchers [1-16] because it is aptomtsing material for device applications [1,2]. GaN crystallizes either in the stable wurtzite ( $\alpha-\mathrm{GaN})$ or metastable zinc-blende $(\beta-\mathrm{GaN})$ structure depending on growth conditions and substrate structure $[5,8,11,12]$. The metastable $\mathrm{GaN}$ polytype has some advantages with respect to the hexagonal one, namely, easy cleavage, smaller bandgap and higher carrier mobilities. $\beta-G a N$ can be grown on various substrates including $\mathrm{GaP}[3,15], \beta-\mathrm{SiC}[5,12,14]$, and $\mathrm{Si}[5,12]$, but the $\mathrm{GaN} / \mathrm{GaAs}$ is of particular interest because GaAs is widely used for opto- and microelectronics. The growth of $\mathrm{GaN}$ on $\mathrm{GaAs}$ is complicated owing to the large lattice mismatch $(-20 \%)$ and the substantially different chemistry of As and $N$ [3,8,13-16]. On the other hand, the large mismatch in lattice parameters between the epilayer and the substrate results in full strain relaxation of the system after completion of the first or second GaN monolayer. The small size and great reactivity of nitrogen atoms relative to As atoms tends to cause deterioration of the GaAs surface: it is not stable under exposure to chemically active nitrogen. Formation of an islanded $\mathrm{GaN}$ layer with a highly facetted interface has been observed with exposure of the GaAs surface to chemically active nitrogen which is a significant fraction of atomic nitrogen produced by an If plasma source. Although interfacial facetting was shown to decrease the defect density in the GaN layer, it is not suitable for device applications. To improve the structure of the $\mathrm{GaN}$ layer while maintaining a flat interface, optimization of the nucleation process is necessary.

The goal of the present study is to better understand how nitrogen reacts with a GaAs (001) surface and elucidate the correlation between interface structure and growth conditions. High resolution electron electron microscopy (HREM) was applied to several GaN/GaAs structures grown under different $\mathrm{Ga}$ fluxes for fixed nitrogen plasma conditions and fixed substrate temperature. The results show that there is an optimal $\mathrm{Ga}$ flux for an otherwise fixed set of nucleation parameters that results in a smooth $\mathrm{GaN} / \mathrm{GaAs}$ interface and relatively low defect density in the GaN epilayer.

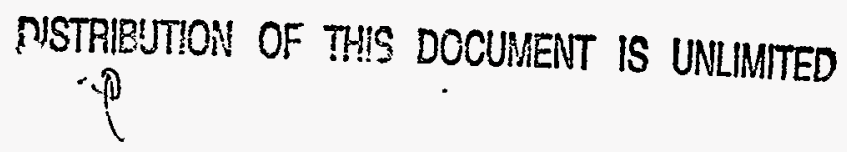




\section{DISCLAIMER}

This report was prepared as an account of work sponsored by an agency of the United States Government. Neither the United States Government nor any agency thereof, nor any of their employees, make any warranty, express or implied, or assumes any legal liability or responsibility for the accuracy, completeness, or usefulness of any information, apparatus, product, or process disclosed, or represents that its use would not infringe privately owned rights. Reference herein to any specific commercial product, process, or service by trade name, trademark, manufacturer, or otherwise does not necessarily constitute or imply its endorsement, recommendation, or favoring by the United States Government or any agency thereof. The views and opinions of authors expressed herein do not necessarily state or reflect those of the United States Government or any agency thereof. 
The growth of 50-100 nm thick GaN layers was carried out using a RIBER 32P molecular beam epitaxy (MBE) system. An Oxford Applied Research MPD21R If plasma source operated at $300 \mathrm{~W}$ with a nitrogen flow of $2 \mathrm{sccm}$ was employed to generate active nitrogen. Optical characterization of the plasma revealed the presence of $\mathrm{N}^{\circ}, \mathrm{N}^{+}, \mathrm{N}^{2+}$, and $\mathrm{N}^{2 *}$. The GaN was deposited at $620^{\circ} \mathrm{C}$ on an n-/n+ GaAs:Sn buffer layer on an $n+\mathrm{GaAs}$ :Si substrate. The buffer layer was grown at $580^{\circ} \mathrm{C}$ on a GaAs (001) substrate using $\mathrm{As}_{4}$ supplied through a valved cracking cell with the cracking zone run at $600^{\circ} \mathrm{C}$ to avoid cracking the arsenic. Upon completion of the buffer layer growth was interrupted while the substrate temperature was adjusted to $620^{\circ} \mathrm{C}$ under an $\mathrm{As}_{4}$ flux. The upper $\mathrm{n}-\mathrm{GaAs}$ layer doped at a level of $2 \times 10^{16} \mathrm{~cm}^{-3}$ had a conventional As stabilized ( $2 \times 4)$ surface reconstruction as observed by reflection high energy electron diffraction (RHEED). After stabilizing the substrate temperature the plasma was initiated behind a closed shutter. Nucleation proceeded by opening the plasma shutter immediately followed by opening the Ga shutter. The As flux remained on for an estimated eight monolayers of the GaN growth. Optimal nucleation was obtained by simultaneously applying equal fluxes of $\mathrm{Ga}$ and active nitrogen to the substrate at the highest possible growth rate. This minimizes nitrogen diffusion into the substrate by burying the interface as rapidly as possible. The $\mathrm{Ga}$ to nitrogen ratio was varied by changing the $\mathrm{Ga}$ flux delivered to the substrate.

$\mathrm{GaN} / \mathrm{GaAs}$ epitaxial layers were characterized by conventional electron microscopy and high resolution electron microscopy using two cross-sections parallel and perpendicular to the "major" flat of the GaAs wafer. Electron microscopy was carried out on JEOL 200CX and Topcon 002B microscopes operated at $200 \mathrm{kV}$.

\section{RESULTS AND DISCUSSION}

1. "Stoichiometric" growth.

The best quality of GaN layers was achieved by "stoichiometric" growth in terms of Ga to $\mathrm{N}$ ratio. The term "stoichiometric" is used here only in the context of nucleation of a good GaN film under these growth conditions. Fig. 1 shows TEM cross-sectional images of nearstoichiometric GaN layer taken perpendicular (a) and parallel (b) to the major flat of the GaAs wafer.

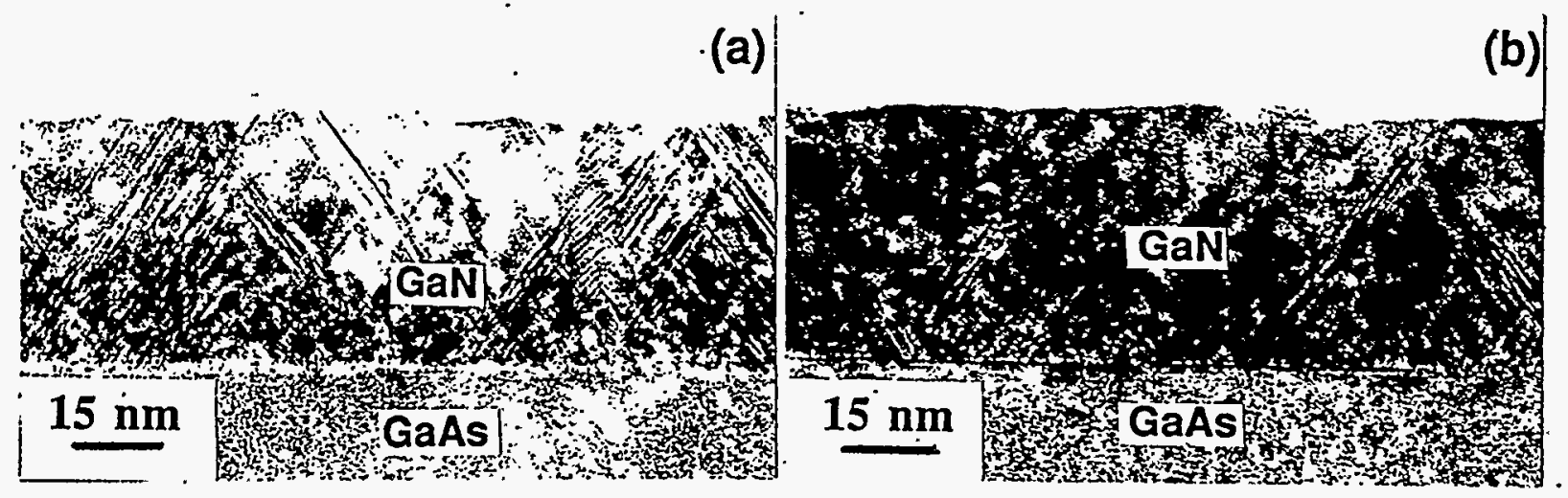

Fig.1, a-b. Near-stoichiometric GaN layer grown on (001) GaAs. TEM cross-sectional images (a) and (b) were taken perpendicular and parallel to the major flat of the $\mathrm{GaAs}$ wafer, respectively.

Interfaces between the GaAs and the GaN were surprisingly abrupt for the samples under study as compared to the samples grown by other groups $[12,13]$. Interface roughness for the "stoichiometric" growth was in the range of 0.8-1.2 $\mathrm{nm}$. Atomic structure of the GaN-GaAs interface is shown in Fig. 2. Because of the high misfit $(f \sim 20 \%)$ in lattice parameters between $\mathrm{GaN}$ and GaAs the interface contains a dense array of misfit dislocations extended in two perpendicular [110] and [-110] crystallographic directions. For $20 \%$ misfit $f$ in lattice parameters 

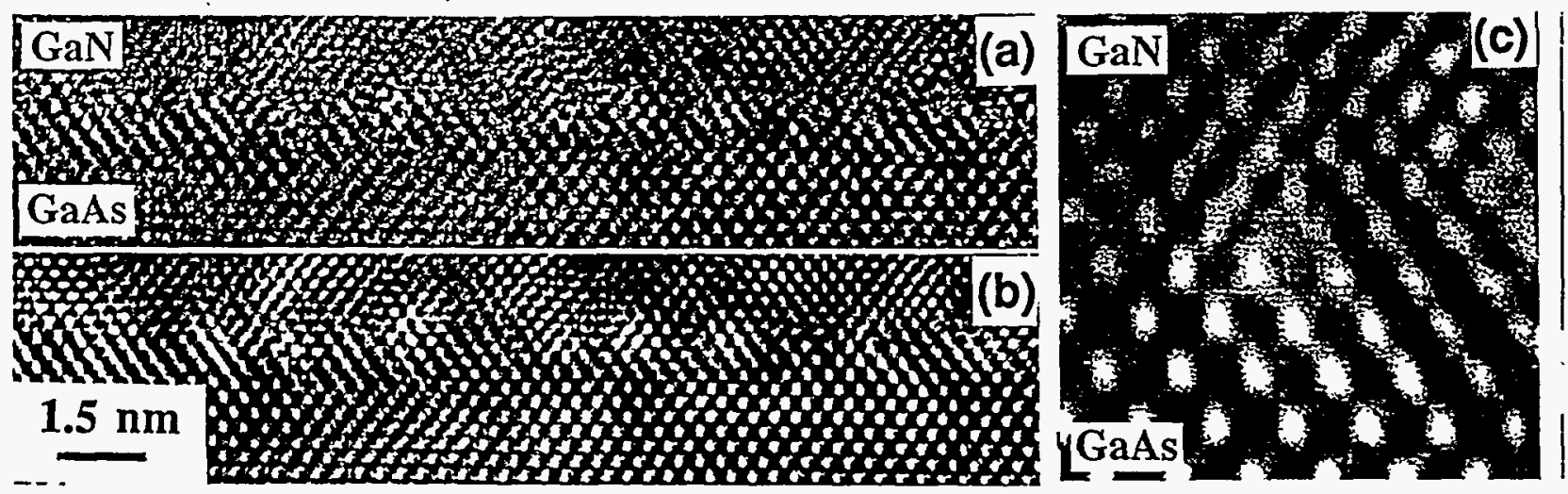

Fig.2, a-c. Atomic structure of the GaN-GaAs interface: cross sectional HREM images before (a) and after (b) image filtering. 90 $0^{\circ}$ Lomer dislocation at GaN-GaAs interface is shown in (c).

For a fully relaxed $\mathrm{GaN}$ layer the equilibrium spacing between $90^{\circ}$ misfit dislocations with Burgers vector $b$ of $1 / 2[110]$ should be $L=b / f \sim 1.6 \mathrm{~nm}$. Most observed dislocations are $60^{\circ}$ dislocations that appear in pairs separated by a few atomic distances. These pairs of $60^{\circ}$ misfit dislocations can be considered as dissociated $90^{\circ}$ Lomer dislocations. About $10 \%$ of the misfit dislocations were undissociated $90^{\circ}$ Lomer dislocations (see Fig. $2 \mathrm{c}$ ). The average distance between misfit dislocations is approximately equal to the equilibrium value so that the GaN layer is almost fully relaxed. However, there is a local strain at the interface that is associated with inhomogenious distribution of the misfit dislocations (fluctuations in dislocation spacing).

While the Burgers vector of a $90^{\circ}$ Lomer dislocation lies in the interface plane, $60^{\circ}$ misfit dislocations have Burgers vectors of $1 / 2[110]$ which are inclined to the interface. Thus, $60^{\circ}$ misfit dislocations have (111) planes as glide planes and can easily dissociate into $30^{\circ}$ and $90^{\circ}$ partials with formation of a stacking fault ribbon between them. Many of the $60^{\circ}$ misfit dislocations are dissociated and, therefore, the GaN layer contains a high density of stacking faults (SFs). This results in streaking of the diffraction spots on electron diffraction patterns (Fig.3).
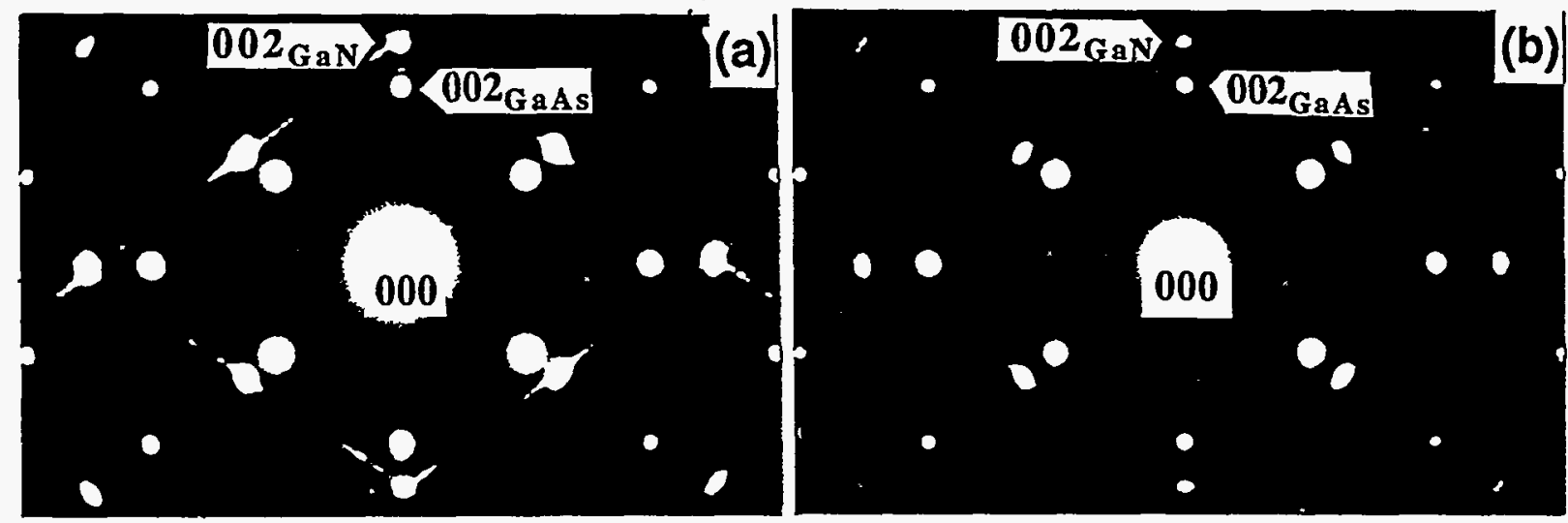

Fig.3, a-b. Selected area diffraction patterns taken perpendicular (a) and parallel (b) to the major flat of the GaAs wafer. Note the different degree of $\mathrm{GaN}$ spot streaking in the figures (a) and (b).

The SF density drasticaily decreases away from the substrate toward the top surface in a $20 \mathrm{~nm}$ thick interfacial layer in agreement with previous observation. However, their density near the top of the GaN layer (Fig.1) is different by an order of magnitude in the two cross-sections 
the top of the GaN layer (Fig.1) is different by an order of magnitude in the two cross-sections taken perpendicular (a) and parallel (b) to the major flat of the GaAs wafer. It is lower in the cross sections taken parallel to the major flat of the GaAs wafer (Fig. 1b). This asymmetry of SF distribution results in different degrees of $\mathrm{GaN}$ spot streaking in the selected area diffraction patterns taken perpendicular (Fig. 3a) and parallel (Fig.3b) to the major flat and in different shapes of (002) $x$-ray reflexions in reciprocal space (Fig.4).

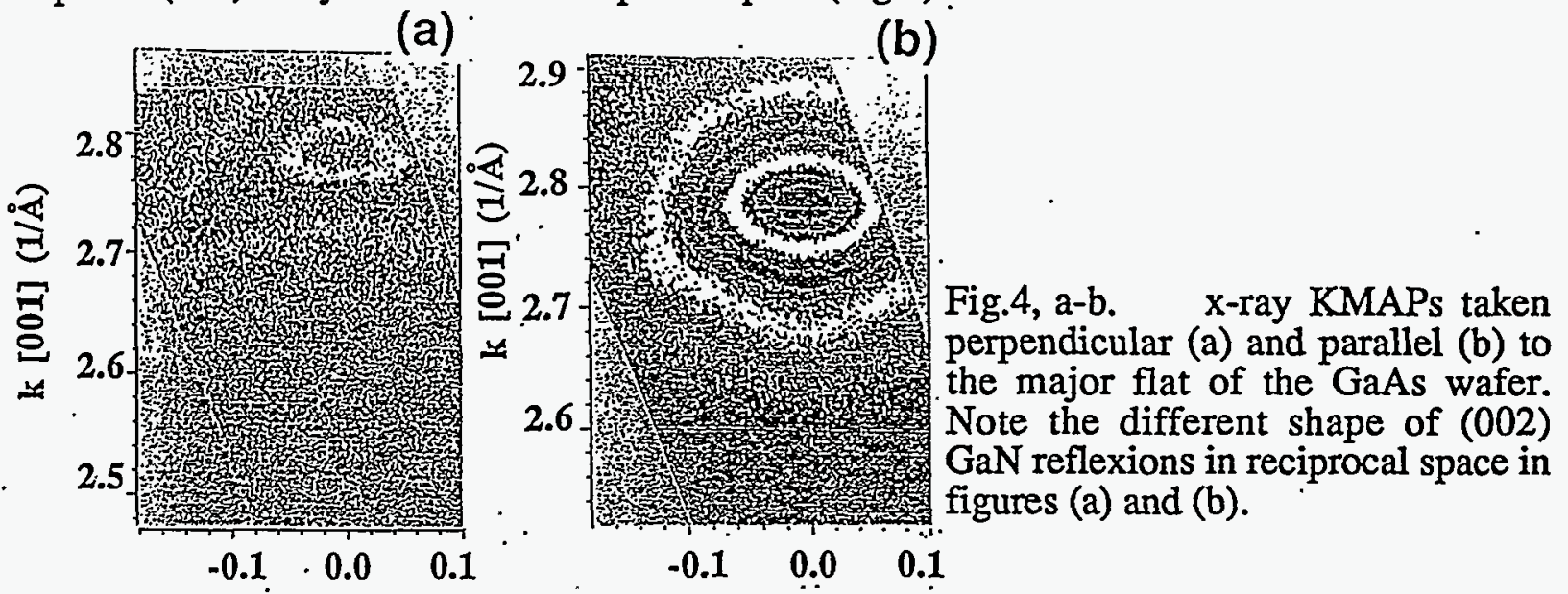

The decrease of stacking fault density results from their annihilation and termination within the GaN layer. Formation of sets of stacking faults is often associated with steps at the $\mathrm{GaN}-\mathrm{GaAs}$ interface so that their density correlates with interface roughness and local strain at the interface. This asymmetry of SF distribution might be associated either with the difference in the atomic structure of surface steps along [1.10] and [-110] crystallographic directions on the (001) GaAs or with different mobilities of $\alpha$ and $\beta$ dislocations and their respective partials. SF density at the interface is only slightly less for the cross section parallel to the major flat while it is less by an order of magnitude for the top of GaN layer in comparison with that of the cross section perpendicular to the major flat. Similarly, an asymmetric defect distribution has been observed for other III-V compounds which was related to the asymmetry of the zinc-blende structure. Our observations suggest that the quality of the GaN layer will increase with thickness for stoichiometric growth conditions.

Near stoichiometric growth was shown to provide the best cubic GaN material [8,13]. However, GaN layers grown. on (001) GaAs at $620^{\circ} \mathrm{C}$ usually had very rough interfaces $[8,13,16]$. In order to. avoid the deterioration of the GaAs surface and, hence, interface roughrning during GaN nucleation, As flux was exposed to the GaAs surface for the first eight monolayers of the GaN growth in the samples under study.

The role of this As flux during the GaN nucleation is not well understood at present, but it is reasonable to assume that As influences the behavior of $\mathrm{Ga}$ adatoms and, hence, their reactions with active nitrogen, resulting in changes. of the density of nucleai. ${ }^{13} \mathrm{Nether}$ in-situ RHEED or ex-situ HREM showed any evidence that As incorporates in the GaN above the solubility limit $(\sim 1 \%)$ during nucleation. High arsenic pressure during the growth has earlier been shown to improve the quality of cubic GaN layers grown on GaAs and GaP substrutes $[3,16]$. On the other hand, As-doping of the GaN layer near the interface might decrease the stacking fault energy making a dissociation of $60^{\circ}$ dislocations more likely.

\section{Microstructure of "Ga-rich" and "Ga-poor" GaN layers.}

Electron microscopy revealed dramatic changes in the structure of the GaN layers with variation of the $\mathrm{Ga}$ flux. Figure 5 shows typical cross-section electron microscopy images of $\mathrm{GaN}$ epilayers grown under Ga-rich conditions in terms of $\mathrm{Ga}$ to $\mathrm{N}$ ratio. The "Ga-rich" layer in Fig.5a has a columnar structure with the lateral size of the grains equal to $15-20 \mathrm{~nm}$. It should not be inferred that $\mathrm{Ga}$-rich growth conditions necessarily lead to the accumulation of metallic $\mathrm{Ga}$ on the surface. Ga-rich nucleation was expected [13] to promote a rough interface because of the high affinity of $\mathrm{N}$ to $\mathrm{Ga}$.

Ga-rich growth leads to multiple twinning and formation of areas of the hexagonal phase 
during the initial stage of $\mathrm{GaN}$ growth in agreement with the results reported earlier [13]. Here, we observed that formation of the hexagonal phase is often associated with change in grain orientation from (001) to (111). due to the twinning (see Fig.5b).
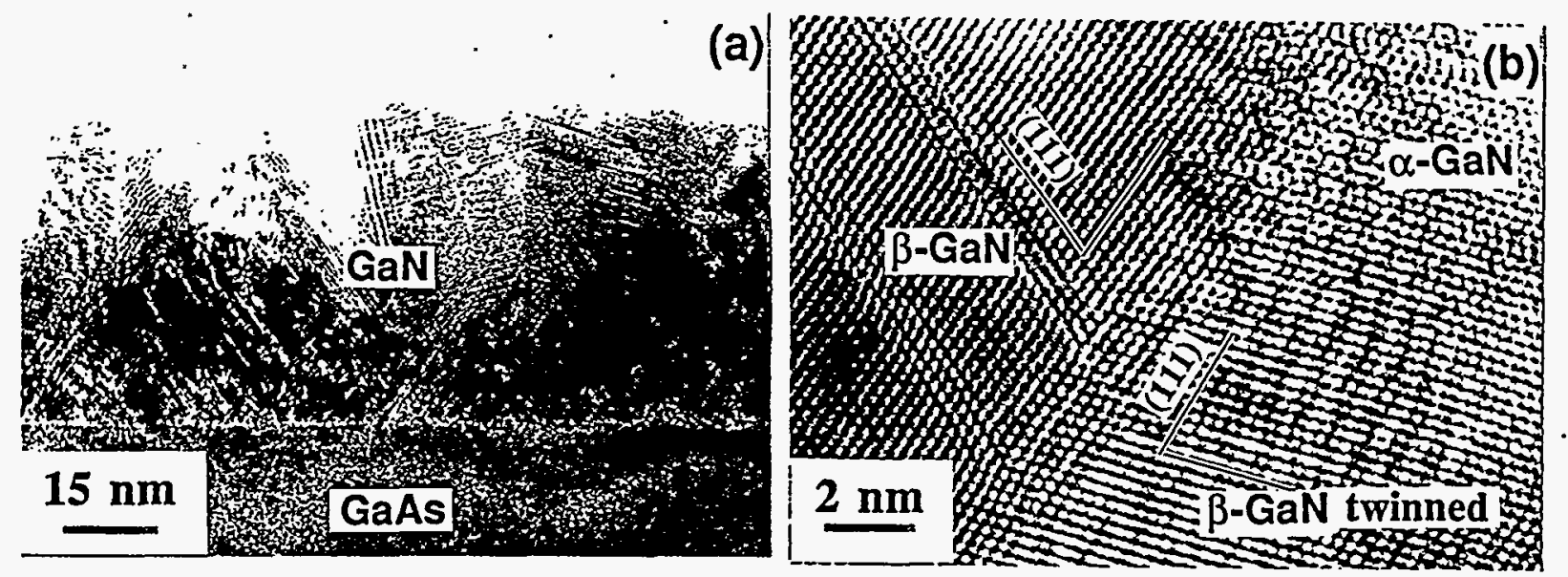

Fig.5. Structure of "Ga-rich" GaN layers grown on (001) GaAs substrate (a). Figure (b) is HREM image showing the formation of the hexagonal GaN phase on (111) planes of the GaN grain in twin orientation.

Figure 6 shows typical cross-section electron microscopy images of GaN epilayers grown under Ga-poor conditions. The $\mathrm{GaN}$ layer contains also contains slightly misotiented grains with amorphous like boundaries between them and a high density of stacking faults. SFs are generated at the interface and at grain boundaries as well.

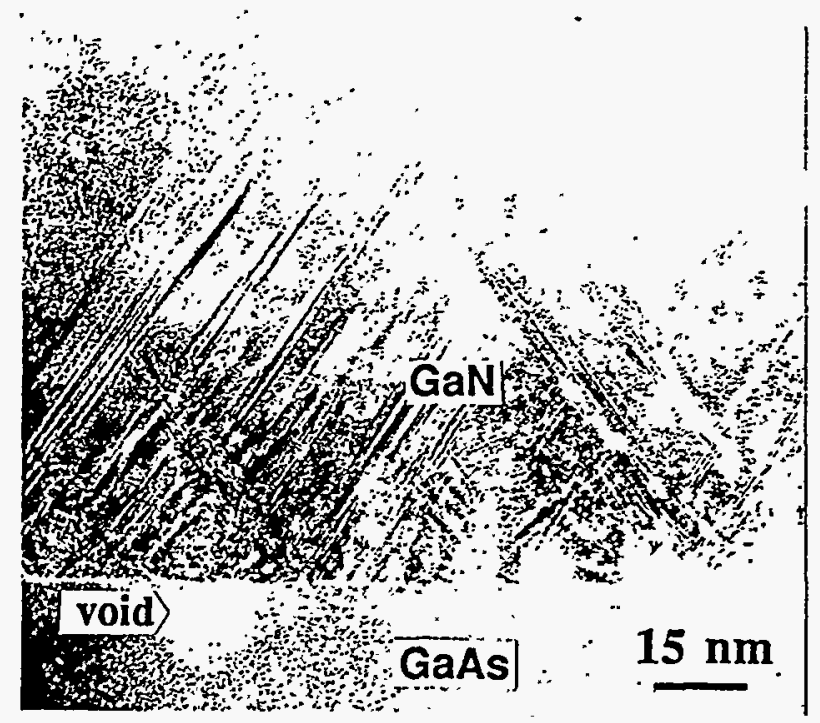

Fig.6. Structure of "Ga-poor" GaN layers grown on (001) GaAs substrate. Note the formation of pockets (voids) in the GaAs substrate below the interface

The twinned cubic and hexagonal grains were also observed at the interface with the substrate for nitrogen rich growth while the top of the GaN layer was cubic (Fig. 6). Formation of those grains was often associated with atomic steps at the interface and with amorphous pockets in the GaAs substrate.

In contrast to the "Ga-poor" layer, grains of $\alpha-\mathrm{GaN}$ in the Ga-rich layers are formed at the interface and grow in columns extending through the layer (Fig.5a). Wurtzite grains are often crystallographically oriented with respect to the GaAs lattice. The predominant orientation relationship between $\mathrm{GaN}$ grains and the GaAs substrate is as follows: $\{0001\}_{\mathrm{GaN}} \|\{001\}_{\mathrm{GaAs}}$, $[1-210]_{\mathrm{GaN}}\left\|[-110]_{\mathrm{GaAs}},[10-10]_{\mathrm{GaN}}\right\|[110]_{\mathrm{GaAs}}$ where the $(-110)$ plane is parallel to the major flat of a GaAs wafer. Hexagonal grains in Fig.5a often tower over the (100) $\beta$-GaN surface by $7 \mathrm{~nm}$ resulting in a rough surface morphology. For nitrogen rich growth the GaN surface is planar to about $\pm 4 \mathrm{~nm}$ and for near stoichiometric growth the surface is planar to about $\pm 1.7 \mathrm{~nm}$. 


\section{CONCLUSIONS}

In conclusion, TEM shows that layer quality depends on the growth conditions particularly the $\mathrm{Ga}$ flux for fixed nitrogen plasma conditions and growth temperature. The highest crystalline quality $\beta-\mathrm{GaN}$ layer was obtained by "stoichiometric" growth while nucleation under Ga-rich conditions resulted in the formation of persistent grains of $\alpha-\mathrm{GaN}$ and grown under very Ga-poor conditions showed grains of $\alpha-\mathrm{GaN}$ near the interface which were subsequently overgrown by the surrounding $\beta-G a N$. Ga-poor samples also showed voids in the $\mathrm{GaN}$ and in the GaAs substrate. The GaN layers contain a high density of stacking faults which drastically decreases toward the GaN surface. Stacking faults are anisotropically distributed in the GaN layer: the majority intersect the interface along lines parallel to the major flat of the GaAs substrate. This anisotropy in SF distribution is likely to be associated with the different atomic structure of $\alpha$ and $\beta$ dislocations in cubic $\mathrm{GaN}$.

\section{ACKNOWLEDGEMENT}

The study at LBNL was supported by the Director, Office of Energy Research, U.S. Department of Energy under Contract No. DE-AC03-76SF00098. The use of facilities of the National Center of Electron Microscopy is greatly appreciated. The work at SNL was supported by the U.S. Department of Energy under contract No. DE-AC04-94AL85000.

\section{REFERENCES}

1. S. Nakamura, M. Senoh, S. Nagahama, N. Iwasa, T. Yamada, T. Matsushita, H. Kiyoku, and Y. Sugimoto, Jpn. J. Appl. Phys. 35, L74 (1996).

2. S. N. Mohammad, A. A. Salvador, and H. Morkoç, Proceedings of the IEEE 83, 1306 (1995).

3. L. C. Jenkins, T. S. Cheng, C. T. Foxon, S. E. Hooper, J. W. Orton, S. V. Novikov, and V. V. Tret'yakov, J. Vac. Sci. Technol. B 13, 1585 (1995).

4. H. Liu, A. C. Frenkel, J. G. Kim, and R. M. Park, J. Appl. Phys. 74, 6124 (1993).

5. T. D. Moustakas, T. Lei, and R. J. Molnar, Physica B 185, 36 (1993).

6. R. C. Powell, N.-E. Lee, Y.-W. Kim, and J. E. Greene, J. Appl. Phys. 73, 189 (1993).

7. M. Kasu, T. Makimoto, and N. Kobayashi, Appl. Phys. Lett. 68, 955 (1996).

8. H. Yang, O. Brandt, M. Wassermeier, J. Behrend, H. P. Schönherr, and K. H. Ploog, Appl. Phys. Lett. 68, 244 (1996).

9. J. Neugebauer, and C. G. van de Walle, In E. A. Fitzgerald, J. Hoyt, K.-Y. Cheng, and J. Bean (Eds.), Strained Layer Epitaxy - Materials, Devices, Processing and Device Applications, 379 (pp. 3). San Francisco, California, USA: Materials Research Society (1995).

10. R. J. Molnar, and T. D. Moustakas, J. Appl. Phys. 76, 4587 (1994).

11. M. Nagahara, S. Miyoshi, H. Yaguchi, K. Onabe, Y. Shiraki, and R. Ito, Journal of Crystal Growth 145, 197 (1994).

12. D. Chandrasekhar, D. J. Smith, S. Strite, M. E. Lin, and H. Morkoç, Journal of Crystal Growth 152, 135 (1995).

13. H. Yang, O. Brandt, and K. Ploog, phys. stat. sol. (b) 194, 109 (1996).

14. H. Okumura, K. Ohta, T. Nagatomo, and S. Yoshida, Journal of Crystal Growth 164, 149 (1996)

15. T. S. Cheng, L. C. Jenkins, S. E. Hooper, C. T. Foxon, J. W. Orton, and D.E. Lacklison, Appl. Phys. Lett. 66, 1509 (1995).

16. C. T. Foxon, T. S. Cheng, S. E. Hooper, L. C. Jenkins, J. W. Orton, D.E. Lacklison, S. V. Novikov, T.B. Popova, and V. V. Tret'yakov, J. Vac. Sci. Technol. B 14, 2346 (1996). 